\title{
Fatores Determinantes para as Expectativas de Primigestas acerca da Via de Parto
}

Primigravid Expectations about the Delivery Method and the Causal Factors for their Choice

Ricardo Porto Tedesco, Nelson Lourenço Maia Filho, Lenir Mathias, Ana Luiza Benez, Valeska Christine Lemes de Castro, Guilherme Muniz Bourroul, Fernando Ivan dos Reis

\section{RESUM0}

Objetivo: conhecer as expectativas de primigestas com relação à via de parto, bem como os motivos de sua escolha, procurando melhorar a qualidade do relacionamento médico-paciente.

Métodos: foi realizado estudo do tipo qualitativo por meio da análise do sujeito coletivo, incluindo primigestas atendidas de setembro a novembro de 2003 nos pronto-socorros dos serviços da Faculdade de Medicina de Jundiai. Foi aplicado questionário especialmente elaborado para responder aos objetivos propostos pela pesquisa o questionário é baseado nas dúvidas apresentadas por pacientes que freqüentaram o serviço meses antes da elaboração do projeto. O consentimento livre e esclarecido, assinado pela gestante e por um dos pesquisadores responsáveis. Foi obtido para fins de padronização da amostra a seleção das pacientes seguiu critérios de inclusão: idade maior que 16 anos, primigestas, que estivessem recebendo assistência pré-natal e consentimento pós-informado lido e esclarecido. Foram considerados critérios de exclusão pacientes mentalmente incapacitadas e gestantes de alto risco com doenças que pudessem interferir na escolha da paciente.

Resultados: a população estudada teve como perfil mais prevalente mulheres com mais de 21 anos, brancas, casadas, com segundo grau completo e que estavam no terceiro trimestre da gestação. A via de parto de preferência da maioria das mulheres (90\%) foi o parto vaginal normal, sendo que as principais justificativas foram: a praticidade para sua realização (94\%) $e$ o medo do sofrimento e dor no pós-parto causados pela cesárea. Encontramos relação entre a preferência pelo parto vaginal com mulheres de maior idade, casadas, não havendo diferença significativa entre as raças.

Conclusão: estes resultados nos mostram enorme contraste entre a preferência das mulheres e os altos índices de cesárea no Brasil. Concluímos que deve haver falha de informação, falta de diálogo entre os profissionais da saúde e a paciente sobre as possíveis dificuldades, dúvidas e anseios que permeiam a escolha por determinada via de parto. Do ponto de vista ético concluímos que os obstetras devem questionar cada indicação para a realização de uma cesárea e respeitar a autonomia da escolha materna sem ignorar os verdadeiros critérios clínicos que levam à decisão médica pela via de parto.

PALAVRAS-CHAVE: Via de parto. Primigesta. Gravidez normal. Parto normal. Cesariana.

Faculdade de Medicina de Jundiaí - Departamento de Tocoginecologia

Correspondência:

Ricardo Porto Tedesco

Rua Francisco Telles, 250

Caixa Postal 1295

13202-550 - Jundiaí - SP

Fone: (11) 4587-1095

e-mail: mtedesco@terra.com.br
Introdução

A gestação representa período único e especial na vida da mulher, no qual a sensação de tornar-se mãe confunde-se muitas vezes com incertezas, medos e inseguranças. Esse fato aflora nas primigestas, especialmente no que se relaciona 
ao momento do parto. Em muitos casos, a escolha da via de parto motiva grande discussão clínica. Em geral, a gestante não participa dessa discussão, sendo, quando muito, informada sobre a decisão médica final. Não se leva em consideração sua aceitação ou não em relação à conduta a ser tomada, nem a associação entre a sua aceitação e os resultados perinatais obtidos.

A aparente "liberdade de escolha" assegurada à mulher é, na prática, freqüentemente ceifada pela manipulação das informações prestadas sobre os riscos envolvidos nos procedimentos do parto $^{1}$. Amu et al. $^{2}$ acreditam que a escolha materna é direito humano fundamental, mas a gestante necessitaria de mais informação. Besio ${ }^{3}$ compartilha desta afirmação. No entanto, mesmo acreditando que a escolha materna reflita autonomia, afirma que a mesma não deva se sobrepor a critério clínico médico quando este sabe qual via de parto é a melhor para a mulher.

Nas últimas décadas tem-se observado aumento global da incidência de cesárea. Em nosso país essa incidência chegou a 36,4\% em 1997 e 27, 1\% em 19994. Em Jundiaí, município do Estado de São Paulo, no ano de 2002, observou-se que de 4966 partos realizados, $57,0 \%$ foram partos vaginais normais ${ }^{5}$. Em 2003, uma das maternidades desse mesmo município, que só presta atendimento a gestantes conveniadas ou particulares, teve incidência de cesárea de 80\%. É importante ressaltar que a OMS considera aceitável incidência de cesárea de $15 \%$. Para Cecatti ${ }^{6}$, o Brasil pode comemorar a perda do recorde de cesarianas para o Chile, creditando a vitória à medida adotada pelo Ministério da Saúde, que fixou há aproximadamente seis anos limite de $40 \%$ nas cesarianas feitas pelo SUS em cada região, deixando de pagar a conta hospitalar do excedente.

Os autores que aceitam o parto cesáreo como opção de escolha materna o fazem por acreditar que a mulher tem o direito de opinar sobre a via de parto ${ }^{7}$. Para os obstetras, os determinantes das altas taxas de cesáreas podem ser classificados em razões culturais, organizacionais e institucionais. Faúndes e Cecatti ${ }^{8}$, ao estudarem a proporção de cesáreas entre os partos assistidos pela previdência social no Brasil em 1991, consideraram as mesmas razões anteriormente citadas, incluindo a questão legal como mais um fator determinante para tais taxas. Osava ${ }^{9}$ compartilha da mesma opinião, acreditando que as representações do parto são estruturadas pelos sujeitos de acordo com a classe social da clientela, estando mais relacionada a um ato "instintivo", para as mulheres das classes populares, e mais relacionada a um ato "cultural" para as mulheres das classes mais elevadas, e que o parto vaginal é fortemente associado à idéia de "imprevisibilidade", ao passo que o parto cesáreo é predominantemente associado à idéia de segurança. De acordo com Berquó $^{10}$, a demanda por laqueadura tubária concomitante à cesárea é também apontada como responsável por essas altas taxas.

Em nossa sociedade, além da laqueadura tubária, outros fatores contribuem para as altas taxas de cesárea: pensamento por parte da mulher de que este tipo de parto é indolor e preserva a anatomia da vagina para as relações sexuais, falta de remuneração da anestesia para o parto normal, falta de acesso da população à informação sobre os riscos das cirurgias obstétricas, conveniência médica e falta de equipamentos adequados para monitorização fetal e materna. Besio ${ }^{3}$ também relaciona o crescente número de cesáreas em quase todo o mundo ao sistema de saúde e judicial, acreditando que os plantões noturnos e os atendimentos de urgência relacionados ao parto vaginal normal aumentam as demandas judiciais por mau resultado neonatal, produzindo no médico a sensação de insegurança e o desconsolo pelo esforço de defender o parto vaginal normal.

Maia Filho et al. ${ }^{11}$ acreditam que o aumento nas indicações de cesáreas, tanto em clínicas particulares como em conveniadas, deve-se à melhoria das condições de cirurgia, de anestesia, antibioticoterapia e hemotransfusão. Porém, associam muito desse aumento também às indicações que não são por doenças obstétricas, salientando ainda a maior demanda de profissionais não habilitados a entrarem no campo da tocoginecologia, com conhecimentos limitados sobre o útero gravídico. Faúndes e Silva ${ }^{12}$ ampliam as justificativas pelas altas taxas de cesárea nos últimos tempos nas escolas médicas. Para estes autores, cada vez menos existe a preocupação das escolas obstétricas em preparar seus alunos para o exercício de boa tocurgia, sendo raros os médicos jovens que sabem avaliar com precisão a bacia obstétrica, o trajeto e identificar sua proporcionalidade. Para eles as escolas médicas não formam adequadamente seus alunos.

Em contrapartida, Paim ${ }^{13}$ firma a idéia de que algumas mulheres entendem as dores do parto vaginal como algo inerente ao processo de parturição, sendo parte da experiência de "tornarse mãe" (passagem para o status de adulta), levando-as à escolha pela via vaginal. Diniz et al. ${ }^{14}$ definiram as dores do parto vaginal como processo de "naturalização do sofrimento". Besio ${ }^{3}$, após enumerar justificativas para as altas taxas de cesárea, defende as razões para que os partos sejam vaginais. Para ele a gravidez e o parto são processos 
naturais que acontecem por si mesmos, não necessitando da intervenção de terceiros. A cesárea seria uma intervenção médica sobre um processo que a natureza resolve e regula sem artifícios. Para Cecatti ${ }^{6}$, as discussões hoje estão mais centradas na escolha do tipo de parto pela mulher, sendo que se a maior parte preferia cesariana porque era vendida como um produto de qualidade, atualmente, estabelecidos o valor do parto normal bem feito e as possiveis complicações da cirurgia, a visão passa a ser mais favorável ao processo natural.

Diante das discussões científicas e sociais sobre as vias de parto que persistem durante décadas, é importante conhecermos a opinião daquela que é a principal envolvida neste processo de transição de uma fase de ousadia e liberdade que marcam a sua vida antes do "tornar-se mãe", para uma fase de compromisso, responsabilidade e obrigações que chegam com a maternidade.

Portanto, teve este estudo como objetivo conhecer melhor o que pensam as mulheres sobre o parto, procurando melhorar a qualidade do relacionamento médico-paciente.

\section{Métodos}

Este estudo foi do tipo qualitativo, por meio da análise do sujeito coletivo, incluindo primigestas atendidas nos pronto-socorros dos Serviços da Faculdade de Medicina de Jundiaí por internos, residentes e professores da Disciplina de Obstetrícia, no período de 20 de setembro a 20 de novembro de 2003. Por se tratar de estudo qualitativo, em que o valor epidemiológico dos dados obtidos está na qualidade das respostas, definiu-se um tamanho amostral de 40 pacientes, acreditandose que aquelas, bem como os motivos pela escolha de determinada via de parto, se repetiriam. Esse fato realmente se confirmou, pois com amostra de cerca de quinze pacientes o conteúdo de suas respostas tornou-se repetitivo.

Foi aplicado um questionário, especialmente elaborado para esse fim, baseado nas dúvidas acerca da via de parto de primigestas atendidas previamente nos serviços. Na ocasião foi obtido um consentimento livre e esclarecido assinado pela gestante e por um dos pesquisadores responsáveis. O questionário elaborado para pesquisa de intenção de parto foi dividido em três partes: parte um, onde constavam características demográficas da entrevistada; parte dois, dados da gestação, e parte três, onde foi abordado, num primeiro momento, a escolha da via de parto, com transcrição das falas das participantes, e numa segunda etapa as vantagens e desvantagens por determinada via, dadas algumas opções. As primigestas foram selecionadas aleatoriamente nos pronto-socorros durante atendimentos por intercorrências da gravidez, nas salas de observação e/ou pré-parto. As mesmas foram esclarecidas sobre a intenção científica da pesquisa, as questões abordadas no questionário e o sigilo de suas respostas, não havendo recusa em participar do estudo.

Foram considerados critérios de inclusão: idade maior que 16 anos, primigestas (gestação única) que estivessem recebendo assistência pré-natal e consentimento pós-informado lido e esclarecido.

Foram considerados critérios de exclusão pacientes mentalmente incapacitadas, gestantes de alto risco, incluindo diabéticas, cardiopatas, pneumopatas, com restrição de crescimento intra-uterino, com polidrâmnio ou oligoâmnio, descolamento prematuro da placenta, placenta prévia, rotura prematura das membranas, malformação fetal e pós-datismo, dentre outras situações clínicas que pudessem interferir na escolha da paciente.

Procurou-se conhecer as relações entre a idade, o estado civil, a escolaridade, a raça e a época da gestação, associando-os à escolha por determinada via de parto.

Este trabalho teve seu projeto aprovado pelas Comissões de Ética dos centros participantes (Faculdade de Medicina de Jundiaí, Hospital Universitário de Jundiaí e Hospital Regional de Franco da Rocha - DIR IV).

\section{Resultados}

Dentre quarenta primigestas estudadas encontramos mulheres com idade entre 16 e 30 anos (28\% com menos de 18 anos, 35\% entre 18 e 21 anos e 37\% com mais de 21 anos), sendo $43 \%$ brancas, $25 \%$ negras e $32 \%$ de outras etnias. Três mulheres estavam no segundo trimestre de gestação e trinta e sete mulheres, no terceiro trimestre. Ao avaliarmos o grau de escolaridade de nossas entrevistadas, observamos que 12 gestantes (30\%) possuíam apenas o primeiro grau completo, 27 $(67,5 \%)$ o segundo grau e $1(2,5 \%)$ o terceiro grau.

A via de parto de preferência da maioria das mulheres foi o parto vaginal normal (PVN), o que correspondeu a $90 \%$ das gestantes estudadas.

Comparando-se a escolha da via de parto com a idade das mulheres, notamos associação da preferência pelo PVN com o aumento da idade (Figura 1). 


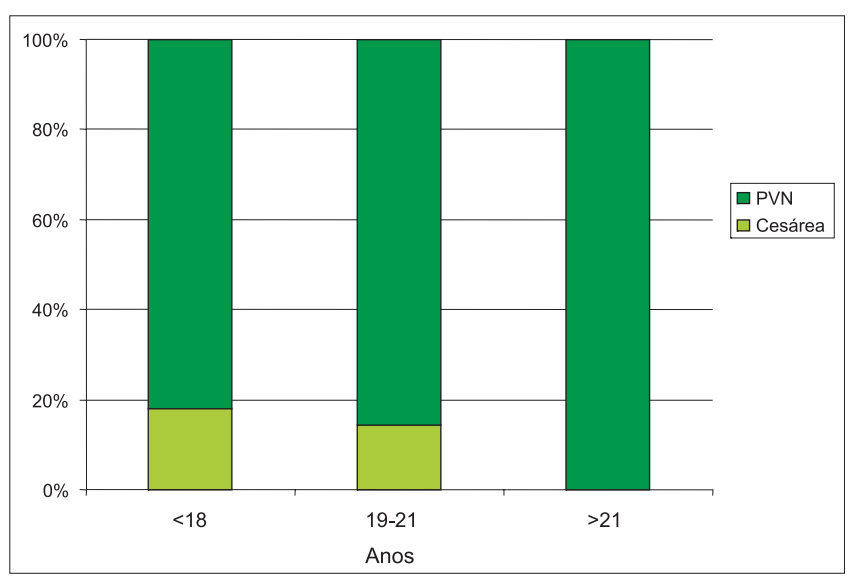

Figura 1 - Escolha da via de parto conforme a faixa etária.

$\mathrm{PVN}=$ parto vaginal normal

Das mulheres que optaram pela cesárea, $75 \%$ eram solteiras. Em contrapartida, esta taxa foi de $22 \%$ naquelas que deram preferência ao parto normal.

Associando a escolaridade à escolha da via de parto observa-se que, dentre as mulheres com primeiro grau completo, $8 \%$ são adeptas ao parto cesáreo, ao passo que nas mulheres com segundo grau completo essa taxa foi de $11 \%$.

Considerando-se a raça, das mulheres de etnia branca, $82 \%$ deram preferência ao PVN, ao passo que $90 \%$ das mulheres de etnia negra o escolheram como via de parto.

Dentre as mulheres que se encontravam no segundo trimestre, não houve nenhuma opção por cesárea. Já naquelas que se encontravam no terceiro trimestre, essa via de parto foi escolhida por $11 \%$ das mulheres.

Quando perguntadas sobre qual procedimento é melhor remunerado na opinião das mulheres, $77 \%$ responderam ser a cesárea.

Ao indagarmos sobre o motivo da escolha pelo parto vaginal, dadas algumas opções como: praticidade deste procedimento, medo do sofrimento e dor do pós-parto cesárea, conforto e segurança para o binômio mãe-filho, melhor estética pela ausência de cicatriz, atrapalha menos a vida sexual é menos doloroso para a mulher, a primeira opção foi escolhida como justificativa por $94 \%$ das mulheres, sendo a segunda opção citada por $89 \%$ delas (Tabela 1).

Ao se questionar sobre a cesárea, 100\% das mulheres adeptas do PVN ressaltaram o pós-parto da cesárea mais doloroso, necessitando inclusive de cuidados especiais; $83 \%$ citou o maior risco para a mulher; $58 \%$ apontou o maior tempo de internação; $42 \%$ citou maiores riscos para o recém nascido (RN) e $11 \%$, inadequação com a vida sexual no pós-parto.
Tabela 1 - Motivo da escolha pelo parto vaginal (permitida mais de uma opção para as pacientes).

\begin{tabular}{ll}
\hline Motivo da escolha pelo PVN & $\%$ \\
\hline Praticidade deste procedimento & 94 \\
Medo do sofrimento e dor do pós-parto cesárea & 89 \\
Conforto e segurança para o binômio mãe-filho & 75 \\
Melhor estética pela ausência de cicatriz & 64 \\
Atrapalha menos a vida sexual & 30 \\
Menos doloroso para a mulher & 19 \\
\hline
\end{tabular}

"Depois do parto dá pra cuidar do bebê"; "Porque a recuperação dói menos"; "É mais saudável para mim e para o bebê"; "É mais saudável que a cesárea"; "Tem menos riscos. Cesárea demora mais para sarar, é mais arriscado"; "Não quero ficar com cicatriz na barriga"; "Porque dói na hora mas depois não tem problema"; "Porque é mais fácil e mais rápido"; "Dor é só na hora". Estas foram transcrições das opiniões de algumas mulheres que preferiram o parto vaginal normal, que endossam os resultados citados acima.

Quando interrogadas sobre os motivos da preferência pelo parto cesáreo, dadas algumas opções como: conforto e o sofrimento reduzido, maior segurança para o RN, razões estéticas, interferência na vida sexual futura e segurança da mulher, a primeira opção foi citada por $100 \%$ das mulheres, sendo que a maior segurança, a questão estética e a preocupação com a vida sexual foram escolhidas por $50 \%$ das entrevistadas (Tabela 2).

Tabela 2 - Motivo da escolha pela cesárea (permitida mais de uma opção para as pacientes)

\begin{tabular}{lc}
\hline Motivo de escolha pela cesárea & $\%$ \\
\hline Conforto e o sofrimento reduzido & 100 \\
Mais seguro para o RN & 50 \\
Razões estéticas & 50 \\
Interferência na vida sexual futura & 50 \\
Segurança da mulher & 25 \\
\hline
\end{tabular}

Ao serem indagadas sobre o parto vaginal normal, $100 \%$ das mulheres adeptas ao parto cesáreo temiam a dor e o risco para o bebê que, segundo elas, o parto vaginal geraria; $75 \%$ temiam os riscos para mulher e $50 \%$ delas apontaram para os problemas na vida sexual que, segundo elas, o PVN propicia.

"Tenho medo da dor no parto normal"; "Tenho medo de não ter força"; "O parto cesáreo é mais rápido"; "Porque não sente tanta dor, apesar de 
doer depois do parto". Estas foram afirmações dadas durante entrevista com mulheres que deram preferência ao parto cesáreo.

Ao relacionarmos a faixa etária das primigestas com as razões para escolha por determinada via de parto, dadas algumas opções, encontramos os seguintes resultados: mulheres com menos de 18 anos de idade e aquelas com mais de 21 anos, fizeram sua opção baseadas na idéia de recuperação mais rápida no pós-parto (Figura 2), enquanto as primigestas entre 19 e 21 anos fizeram sua escolha acreditando numa maior segurança para o RN (Tabela 3).

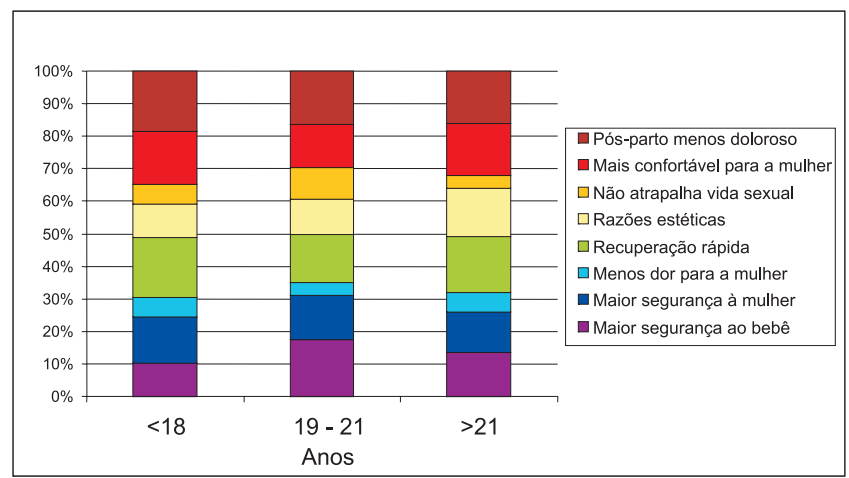

Figura 2 - Razões para escolha de parto conforme a faixa etária.

Tabela 3 - Motivos necessários para realização de cesárea segundo opinião das primigestas (permitida mais de uma opção para as pacientes).

\begin{tabular}{lc}
\hline Opções dadas às primigestas & (\%) \\
\hline Risco para o bebê & 100,0 \\
Quando não há alternativa & 87,5 \\
A mulher tem doença grave & 85,0 \\
O bebê está em posição errada & 95,0 \\
A gravidez passou do tempo & 92,5 \\
A mulher não tem dilatação & 87,5 \\
A mulher fará laqueadura & 30,0 \\
\hline
\end{tabular}

Quanto à necessidade de se realizar cesárea, $50 \%$ das mulheres que deram preferência a este tipo de parto acreditam ser esta necessária em todas as situações propostas no questionário, ao passo que esta porcentagem foi de apenas $25 \%$ no grupo das mulheres que deram preferência ao parto normal.

Dos motivos necessários para realização de cesárea, dadas algumas opções, segundo opinião das primigestas, o risco para o $\mathrm{RN}$ foi citado por todas elas, sendo a opção referente a não haver outra alternativa, exceto a cesárea, a segunda mais escolhida, representando $87,5 \%$ das opiniões das gestantes.

\section{Discussão}

O Brasil apresenta uma das mais elevadas incidências de cesárea de todo o mundo. Essas altas taxas existem por indicações como: preferência da mulher, escolha do profissional e como método de esterilização definitiva ${ }^{15}$.

Segundo Rattner ${ }^{16}$, estima-se que $28,5 \%$ das mortes maternas não ocorreriam se as taxas de cesáreas fossem reduzidas. Faúndes e Silva ${ }^{12}$, ao descreverem o equilíbrio entre o parto vaginal e a cesárea na assistência obstétrica atual, afirmaram que em condições semelhantes o parto cesáreo relaciona-se de 2 a 11 vezes a mais com o risco de morte materna que o parto vaginal normal. O Conselho Regional de Medicina ${ }^{17}$ ratifica tal informação, ao afirmar que as cesáreas realizadas sem consistência na indicação podem ser encaradas como procedimentos desnecessários e que impõem riscos aumentados tanto na morbidade quanto na mortalidade materna. O obstetra deve estar qualificado para assistir ao trabalho de parto com toda segurança do bem-estar fetal e apto a terminá-lo de forma conveniente, evitando a realização da cesárea por insegurança nas suas capacitações em permitir o parto vaginal.

Cotzias et al. ${ }^{7}$ enfatizam que o principal argumento atualmente utilizado pelos que defendem o parto cesáreo como opção é que a escolha pode ser da mulher, e que ela tem o direito de decidir qual a via de parto de sua preferência. Caberia ao médico somente aceitar a decisão de sua cliente. Este argumento supõe que a grande maioria das mulheres preferiria a cesárea ao parto vaginal e o médico simplesmente acataria tal decisão, não valorizando as suas reais indicações.

Faúndes e Silva ${ }^{12}$, com o propósito de destacar a diferença entre a indicação precisa e oportuna de cesárea e os casos em que o seu uso pode trazer mais prejuízos que benefícios para o binômio mãe-filho, analisaram nova classificação das cesáreas, segundo justificativas comumente encontradas nos últimos tempos. Segundo os autores, as cesáreas poderiam ser divididas em: absolutamente não justificadas, cesáreas mal justificadas, com justificação duvidosa e plenamente justificadas. Dentro do primeiro grupo, encontramos as cesáreas com hora marcada com o objetivo de atender a comodidade e conveniência da mãe e/ou do médico e também a oportunidade para realização de laqueadura tubária. Para os autores estas indicações elevam significativamente os riscos de morbidade e/ou mortalidade do binômio. No segundo grupo, das cesáreas mal justificadas, incluem-se antecedente obstétrico de cesárea 
anterior sem se realizar prova de trabalho de parto, ou resolução do parto de apresentação pélvica em multíparas com feto não macrossômico. Nestes casos, os autores acreditam que as condutas obstétricas não são apropriadas, e que portanto aumentam os riscos da mãe sem vantagens para o produto. No terceiro grupo encontram-se as justificativas de distócia funcional, sofrimento fetal agudo e desproporção céfalo-pélvica, cuja efetivação para os autores, aumenta o risco da mulher e do feto sem vantagens para ambos. No último grupo, das cesáreas plenamente justificadas, encontramos aquelas que permitem preservar a saúde ou proteger a vida da mãe e/ou feto, com risco para o binômio menor que aquele gerado pelo prolongamento indevido da gravidez ou pela tentativa de ultimar o parto via transpélvica. Segundo os autores, são elas: placenta prévia centro-total, sofrimento fetal confirmado e intratável, apresentações anômalas e doenças maternas ou do concepto que não permitam a atividade uterina sem agravos pronunciados à saúde da mãe ou do feto. Em outro momento, Faúndes e Perpetuo ${ }^{18}$, ao analisarem a questão ética em relação à indicação do parto cesárea, utilizaram pesquisa realizada pelas Universidades do Texas, Estadual de Campinas (Unicamp), Federal do Rio Grande do Sul, Federal do Rio Grande do Norte e Federal de Minas Gerais, cujos resultados foram preocupantes. Das realizações de cesáreas verificou-se que apenas $13 \%$ tinham real indicação e em apenas 29\% dos casos a razão foi abertamente não médica. Em quase $60 \%$ dos casos a razão "médica" apresentada não justificava a conduta adotada ou era no mínimo duvidosa.

Potter et al. ${ }^{19}$, Hopkins ${ }^{20}$ e Osis et al. ${ }^{21}$ consideram que o grande número de cesáreas não corresponderia à realidade, se as mulheres fossem ouvidas e esclarecidas sobre os procedimentos a serem realizados.

O resultado desse estudo está em conformidade com esses autores, uma vez que, de quarenta gestantes ouvidas, trinta e seis (90\%) têm preferência pelo PVN e somente quatro (10\%) escolheram a cesárea.

Pode-se observar que quanto maior a idade, maior a preferência pelo parto vaginal normal, revelando maior ponderação e reflexão sobre as conseqüências de uma ou outra via de parto, com o amadurecimento da mulher. Pelloso et al. ${ }^{15}$ encontraram em sua pesquisa maior preferência pelo parto cesáreo em mulheres mais jovens, assim como em nosso estudo. Quanto ao grau de instrução, encontramos aumento da preferência pelo parto cesáreo nas mulheres com nível de escolaridade mais elevado. Isto pode ser explicado pelo fato de as mulheres com nível maior de escolaridade exercerem atividade extradomiciliar. Ao optarem pelo parto cesáreo, poderiam determinar a data e a hora para sua realização, não comprometendo suas atividades profissionais.

Em relação ao estado civil, encontramos maior número de mulheres casadas no grupo das que deram preferência ao PVN. É importante enfatizar que o apoio emocional do parceiro durante o parto e o puerpério é fundamental para a mulher, inclusive como suporte emocional durante as dores do parto.

Quanto ao período gestacional, a preferência das primigestas que se encontravam no terceiro trimestre com opção pelo parto cesáreo (11\%) pode revelar o aparecimento das sensações de medo e ansiedade que voltam a surgir no final deste período, estando o parto iminente, fazendoas acreditar que a cesárea traz maior segurança para o RN.

Ao analisarmos o tipo de parto mais fácil para o médico, do ponto de vista das mulheres, pudemos observar que não houve relação com a escolha da cesárea, uma vez que, das 6 mulheres que consideraram a cesárea mais fácil (para o médico), apenas uma escolheu aquela.

Embora trinta e três gestantes (77\%) tenham a idéia de que o parto cesáreo é procedimento melhor remunerado para o médico, a opção pelo PVN prevaleceu.

Não podemos expandir nossos resultados quanto à preferência por PVN ou cesárea para um nível nacional, pois nossa amostra não foi socioeconômica e culturalmente heterogênea como a população brasileira; no entanto, nosso trabalho parece comprovar os resultados do estudo do Professor Joseph Potter, da Universidade do Texas, realizado pelo Núcleo de Estudos da População (NEPO) da Unicamp ${ }^{6}$, que afirmou que não obstante o extrato socioeconômico das regiões do Brasil e o tipo de convênio, aproximadamente $80 \%$ das mulheres em seu estudo expressam o desejo de ter parto normal, o que talvez pudesse ser extrapolado como atitude global.

Neste estudo, as gestantes entrevistadas mostraram nítida preferência pelo parto vaginal. São gestantes atendidas em serviços públicos, o que, em nosso país, em geral ocorre com famílias de baixa renda. Se estas mulheres, teoricamente menos informadas, dão preferência ao parto vaginal, é coerente se pensar que as mulheres mais esclarecidas também tenham a mesma opinião. Por que então a ocorrência de incidências de cesáreas tão elevadas entre mulheres com nível socioeconômico mais elevado?

O medo pelo parto vaginal e suas conseqüên- 
cias ainda pode ser encontrado entre muitas mulheres. Acreditamos que este fato decorre da falta de informação, de diálogo e de uma conversa aberta entre os profissionais e a paciente sobre as dificuldades, dúvidas e anseios.

Conclui-se que são necessárias ações especiais desempenhadas por médicos, enfermeiros e outros profissionais de saúde durante o pré-natal, por meio de orientações e informações, visando diminuir a ansiedade e a insegurança, além de proporcionar maior relação interpessoal entre profissional de saúde e paciente. Para isso é de suma importância a criação de atividades educativas em grupo e participação das mulheres nos programas de preparo ao parto nos serviços de atendimento básico.

Estes resultados nos mostram enorme contraste entre a preferência das mulheres e os altos indices de cesárea no Brasil, a ponto de acreditarmos que a cesárea pode ser considerada epidemia desnecessária. Acreditamos que melhor entendimento dos motivos subjetivos que valorizam tanto a cesárea quanto o PVN possibilitará um exercício consciente da escolha pela via de parto tanto por médicos como pelas mulheres.

Quanto às escolas médicas, é essencial quebrar a resistência cultural, começando por mudança na formação do estudante de Medicina, através de seus mestres, ao retomarem os ensinamentos da tocurgia. Do ponto de vista ético, cabe ao médico questionar cada indicação para a realização de uma cesárea, buscar o aprimoramento e destreza nos procedimentos obstétricos, conhecer melhor a fisiologia do trabalho de parto e do próprio parto e ajustar sua conduta aos imperativos da ética no exercício da profissão médica.

\section{ABSTRACT}

Purpose: to find out the preference in regard to the way of delivery among primigravidae, as well as the reasons for their choice, in order to improve the quality of the doctorpatient relationship.

Methods: a qualitative-type study was conducted through analysis of the collective subject, including primigravidae attended from September to November 2003 at the emergency rooms of the hospital of the "Faculdade de Medicina de Jundiaî". A questionnaire, specially developed to accomplish the proposed objectives was applied. An informed and free consent, signed by the pregnant woman and one of the researchers in charge was obtained. This questionnaire was based on doubts of patients attended at this hospital some months before the trial. For the purpose of sample standardization, the patients' selection followed some inclusion criteria: age above 16, primigravidae that were receiving prenatal assistance and a post-informed and free consent. Mental disorders and clinical and/or obstetric pathologies that could interfere in the patient's choice were considered exclusion criteria.

Results: the studied population had as prevailing profile women in the third quarter of gestation, above 21 years of age, white, married and with completed school. Most of the women (90\%) preferred vaginal delivery for the following main reasons: ease to be done (94\%) and the fear of suffering and pain during the postpartum period caused by cesarean section. There was a relationship between older and married women and the preference for vaginal delivery, with no significant difference between races.

Conclusion: these results show an enormous contrast between women's preference and the high cesarean section rates in Brazil. We conclude that there may be a lack of information and dialogue between the health professionals and patients about the possible difficulties, doubts and anxieties that involve the women's choice for a specific way of delivery. From an ethical point of view, we conclude that obstetricians should question every cesarean section indication and take into account the women's right to choose, without ignoring clinical criteria, when making the medical decision about the way of delivery.

KEYWORDS: Way of delivery. Primigravid. Normal pregnancy. Normal delivery. Cesarean section.

\section{Referências}

1. de Mello e Souza C. C-sections as ideal births: the cultural constructions of beneficence and patient's rights in Brazil. Camb Q Healthe Ethics 1994; 3:358-66.

2. Amu O, Rajendran S, Bolaji II. Should doctors perform elective caesarean section on request? Maternal choice alone should not determine method of delivery. BMJ 1998; 317:463-5.

3. Besio M. Cesárea versus parto vaginal: una perspectiva ética. Rev Med Chile 1999; 127:1121-5.

4. Belisan JM, Althabe F, Barros FC, Alexander S. Rates and implications of caesarean sections in Latin America: ecological study. BMJ 1999; 319:1397-400.

5. Ministério da Saúde. Indicadores Municipais de Saúde. Portal da Saúde. [Acesso: 2004 set 30]. 2004. Disponivel em: http://www.datasus.gov.br

6. Cecatti JG. Brasil comemora perda de um recorde: o de cesarianas [Depoimento a Isabel Gardenal]. Jornal da Unicamp. 2002 set 2-8; 188:9.

7. Cotzias CS, Paterson-Brown S, Fisk NM. Obstetricians say yes to maternal request for elective cae- 
sarean section: a survey of current opinion. Eur J Obstet Gynecol Reprod Biol 2001; 97:15-6.

8. Faúndes A, Cecatti JG. A operação cesárea no Brasil: incidência, tendências, causas, conseqüências e propostas de ação. Cad Saúde Pública 1991; 7:150-73.

9. Osava RH. Estudo das representações sociais do parto entre enfermeiras de maternidade do município paulista [dissertação]. Ribeirão Preto: Univ. São Paulo; 1990.

10.Berquó E. Brasil, um caso exemplar. Anticoncepção e partos cirúrgicos: à espera de uma ação exemplar. Rev Estud Fem 1993; 1:366-81.

11. Maia Filho NL, Mathias L, Hiar J, Pientznauer R, Carvalho, PM. Operação cesariana - A propósito de suas indicações. J Bras Ginecol 1994; 104:423-6.

12.Faúndes A, Silva JLP. O equilíbrio entre o parto vaginal e a cesárea na assistência obstétrica atual. Femina 1998; 26:669-73.

13.Paim HHS. Marcas no corpo: gravidez e maternidade em grupos populares. In: Duarte LE, Leal O, organizadores. Doença, Sofrimento e Perturbação: perspectivas etnográficas. $1^{\mathrm{a}}$ ed. Rio de Janeiro: Editora Fiocruz; 1998. p. 31-47.

14.Diniz SG, Mello-e-Souza C, Portella AP. Not like our mothers. Reproductive choice and the emergence of citizenship among Brazilian rural workers, domestic workers and house wives. In:
Petchesky RP, Judd K, editors. Negotiating Reproductive Rights. Women's Perspectives Across Countries and Cultures. $1^{\text {st }}$ ed. New York: Zed Books; 1998. p. 31-68.

15.Pelloso SM, Panont KT, Souza KMP. Opção ou imposição! Motivos da escolha da cesárea. Arq Ci Saúde Unipar 2000; 4:3-8.

16.Rattner D. Cesárea: bem de consumo? In: $48^{\mathrm{a}}$ Reunião Anual da Sociedade Brasileira para o Progresso da Ciência; 1996 jul; São Paulo, Brasil. São Paulo: SBPC; 1996. p. 339-42.

17. Conselho Regional de Medicina do Estado de São Paulo. Ética em ginecologia e obstetrícia. $2^{\text {a }}$ ed. São Paulo: Cremesp; 2002. p. 90-3. (Cadernos Cremesp).

18.Faúndes A, Perpetuo IHO. Cesárea por conveniência e a ética médica. Ser Med 2002; 19:32-4.

19.Potter JE, Berquó E, Perpetuo IHO, et al. Unwanted caesarean sections among public and private patients in Brazil: prospective study. BMJ 2001; 323:1155-8.

20.Hopkins K. Are Brazilian women really choosing to deliver by cesarean? Soc Sci Med 2000; 51:725-40.

21.Osis MJ, Pádua KS, Duarte GA, Souza TR, Faúndes, A. The opinion of Brazilian women regarding vaginal labor and cesarean section. Int $J$ Gynaecol Obstet 2001; 75:S59-66.

Recebido em: 13/8/2004 Aceito com modificações em: 17/11/2004

\title{
$7^{\circ}$ Congresso da Rede Latino Americana de Reprodução Assistida
}

\section{8 a 10 de abril de 2005}

\section{Ilha de Itaparica - BA}

\section{Realização: Rede Latino Americana de Reprodução Assistida}

\author{
Informações: \\ Tel: (16) 612-4425 \\ E-mail: lmeventos@lmeventos.com.br \\ Home Page: www.lmeventos.com.br
}

